\title{
MEASUREMENT OF PLATELET P-SELECTIN EXPRESSION BY FLOW CYTOMETRY IN PATIENTS WITH ACUTE ISCHEMIC STROKE
}

\author{
Kalmarova $\mathrm{K}^{1}$, Kurca $\mathrm{E}^{1}$, Nosal $\mathrm{V}^{1}$, Dluha $\mathrm{J}^{1}$, Ballova $\mathrm{J}^{1}$, Sokol $\mathrm{J}^{2}$, Skornova $\mathrm{I}^{2}$, \\ STASKo $\mathrm{J}^{2}$, Zolkova $\mathrm{J}^{2}$, VADELOVA L. ${ }^{2}$
}

${ }^{1}$ Clinic of Neurology, Jessenius Faculty of Medicine in Martin, Comenius University in Bratislava, Martin, Slovak Republic

${ }^{2}$ National Center of Hemostasis and Thrombosis, Department of Hematology and Transfusiology, Jessenius Faculty of Medicine in Martin,Comenius University in Bratislava, Martin, Slovak Republic

\begin{abstract}
Aims: The aim of this study was to asses the platelet activation in the acute phase of ischemic stroke and transient ischemic attack (TIA) by defining p-selectin (CD62) expression by flow cytometry in vivo - without stimulation with agonists. We also studied whether antiplatelet therapy supresses the levels of baseline p-selectin expression and verified if there is a correlation between platelet CD62 expression and the type of ischemic stroke.

Methods: We determined the expression of platelet surface p-selectin using whole-blood flow cytometry within the first 48-hours after onset of cerebral symptoms in patients with atherothrombotic and lacunar ischemic stroke and in healthy volunteers. We studied the realationship between antiplatelet medication and the type of ischemic stroke to baseline p-selectin expression.

Results: Patients with acute cerebral ischemia have an excess of circulating platelets that express p-selectin, compared to healthy volunteers. The difference between average p-selectin expression in the group of healthy volunteers and the group of patients with stroke was statistically significant (p-value $<0,000001$ ). Patients with stroke without antiplatelet medication showed a higher p-selectin expression than patients with antiplatelet medication (ASA, CLP, or ASA and CLP), hovewer, the difference was not statistically significant. There is no relationship between CD62 expression and the type of stroke.

Conclusions: We can conclude that p-selectin is a highly sensitive blood biomarker of increased platelet activation. Antiplatelet therapy suppresses baseline p-selectin expression only minimally, insignificantly according to our results.
\end{abstract}

Key Words: flow cytometry, activated platelets, ischemic stroke, p-selectin

\section{INTRODUCTION}

Platelet activation plays a key role in arterial thrombogenesis and, therefore, in the pathophysiology of ischemic stroke $(1,2)$. Accordingly, antiplatelet therapy plays a crucial role in primary and secondary prevention of ischemic stroke. It remains unclear whether this applies to all subtypes, especially lacunar infarcts which differ from atherotrombotic infarction in their etiopathogenesis (3). According to some authors, platelet activation appears to be less important in the pathogenesis of lacunar stroke and efficacy of antiplatelet or anticoagulant therapy remains uncertain (4).

Several studies showed that patients with acute cerebral ischemia have an excess of circulating platelets that express p-selectin (CD62) $(3,5,6,7,8,9)$. P-selectin is an activationdependent platelet receptor, it means it is expressed exclusively during platelet activation.

Address for correspondence:

Vladimír Nosál, Clinic of Neurology, Jessenius Faculty of Medicine in Martin, Comenius University in Bratislava, Martin, Slovak Republic, Kollarova 2, 03659 Martin, Slovak Republic

Phone: +421905 764 991; e-mail: vnosal@gmail.com 
In the resting state it is stored in membranes of the $\alpha$-granule of platelets and in the endothelial cells. Together with E- and L-selectin it constitutes the selectin family. These transmembrane proteins have continued to attract great interest as they support rapid and reversible cell adhesion in flow systems and, thus, play an essential role in multicellular interactions during thrombosis and inflammation. The level of p-selectin inside platelets is independent of that produced by endothelial cells. The expression of circulating soluble (plasma) p-selectin depends on both cell types (endothelial cells and platelets) (6, 10, 11, 12). For diagnostic purposes, p-selectin can be detected on the platelet surface by flow cytometry and by ELISA as a soluble ligand in the plasma (10). Increasing numbers of published studies have attempted to use whole-blood flow cytometry as a platelet function test in clinical settings rather than preparation PRP (platelet rich plasma) $(13,14,15,16)$. Whole-blood flow cytometry has the advantage of directly analyzing individual platelets with a high degree of sensitivity in their native milieu and with minimal artifactual platelet activation $(14,16,17)$.

In platelets, activation is driven by stimulation by vascular agonists such as thrombin, collagen, adenosine diphosphate (ADP), thromboxane A2, and its mimetics and inflammatory agonists including interleukin-4. P-selectin analysis can be performed in non-activated samples, without adding specific agonists, which represents the 'baseline' CD62P positivity in the current in vivo platelet activation status. Platelet reactivity can be also evaluated with CD62P levels on stimulated platelets by using submaximal concentrations of agonists. One agonist is arachidonic acid (AA) the effects of which are blocked by aspirin (aspirin test). The clopidogrel test detects whether P2Y12 antagonists such as clopidogrel, prasugrel, and ticagrelor prevent ADP stimulation of platelets $(18,19)$. Platelet p-selectin values are expressed as a percentage of antibody-positive platelets ( $\mathrm{p}$-selectin positive platelets). In our study we determined the expression of $\mathrm{p}$-selectin in vivo, without stimulation with agonists.

\section{METHODS}

\section{Study population}

We performed a prospective study and enrolled 55 patients with TIA (Transient Ischemic Attack) or ischemic stroke during the acute phase of brain ischemia. There were 33 males and 22 females in the age of 45-87 years, in the average of 61 years. Patients with atherothrombotic and lacunar stroke and TIA were included according to the TOAST classification. Patients with cardioembolic etiology of stroke and TIA were excluded. We defined 2 groups - untreated patients: patients who were not taking antiplatelet medication at the time of occurance of the stroke symptomps $(n=12)$ and treated patients: patients who were taking antiplatelet medication at the time of occurance of the stroke symptomps: acetylsalicylic acid (ASA) $(n=24)$, clopidogrel (CLP) $(n=2)$, and dual antiplatelet therapy (ASA + CLP) ( $n=17)$. The control group consisted of human blood samples obtained from healthy volunteers $(n=50)$. The healthy volunteers were not taking any medications and they did not have history of cerebrovascular or cardiovascular diseases. There were 31 males and 19 females in the age of 19-59 years, in the average of 34.51 years. We used anti-p-selectin and anti-platelet glycoprotein IX (CD 42a) antibodies for whole-blood flow cytometry in order to detect activated platelets in these patients. Values less than $6 \%$ were considered as normal and values $6 \%$ and higher were considered as increased. We assessed the effects of antiplatelet agents to baseline p-selectin expression. Furthermore, we divided patients according to the type of ischemic stroke: patients with lacunar stroke $(n=28)$ and patients with atherothrombotic stroke $(n=27)$. We studied whether there is a correlation between platelet CD62 expression and the type of ischemic stroke. Blood samples were obtained 0-2 days after occurance of stroke. Written informed consent was obtained and the local ethics committee approved this study. 


\section{MATERIALS}

Blood samples were collected into citrate anticoagulated vacutainer tubes and p-selectin was analyzed by whole-blood flow cytometry using a blue laser with a wavelength of 488 $\mathrm{nm}$. Fluorochrome-conjugated anti-CD42a/FITC (fluorescein isothiocyanate conjugated) and anti-CD62P/PE (R-phycoethrin) monoclonal antibodies were used for direct two-colour surface immunophenotyping. The samples were incubated for 10 minutes at room temperature in the dark. Platelet glycoproteins were analyzed by flow cytometer BD FACSVerse ${ }^{\mathrm{TM}}$. Quadrant statistics was used to calculate the relative proportion of antibody-positive platelets - activated cells.

\section{STATISTICS}

The data were analysed using statistical software STATISTICA and the Matlab system. Descriptive statistics were used to describe and summarize the variables in each of the study groups. Fisher exact test was used to compare proportions between groups. Differences between groups were considered statistically significant if the p-value was less than 0.05. A twosided exact Chang-Zhang interval was used to calculate the $95 \%$ confidence interval for the difference of two binomial parameters. The odds ratio was $95 \%$ confidence interval.

\section{RESULTS}

The average p-selectin expression in the group of patients with stroke vs. control group was $10.2868 \%$ vs. $3.146 \%$ (Table 1). In the control group the expression of p-selectin was less than $6 \%$ in 47 patients (94\%). The difference between control and patients groups was statistically significant (p-value $<0.000001)$.

Tab. 1 The average, median, standard deviation (SD), minimum, maximum and range of p-selectin expression in the control group and in patients with stroke

\begin{tabular}{|l|c|c|c|c|c|c|}
\hline & Average (\%) & Median & SD & Minimum & Maximum & Range \\
\hline $\begin{array}{l}\text { P-selectin in patients with } \\
\text { stroke n = 55 }\end{array}$ & 10.2868 & 7.91 & 7.63038 & 0.81 & 30.35 & 29.54 \\
\hline $\begin{array}{l}\text { P-selectin in control group } \\
\mathrm{n}=50\end{array}$ & 3.146 & 2.5 & 2.07229 & 0.3 & 12.0 & 11.7 \\
\hline
\end{tabular}

Increased p-selectin expression in the group of untreated patients was present in 10 of 12 patients $(83.33 \%)$. In the group of treated patients p-selectin expression was increased in a smaller number of patients - 26 of 43 patients (60.47\%). The difference between these two groups was not statisticaly significant (p-value $=0.1830 ;$ OR $+95 \%$ CI= 3.962 (0.6363-16.7970)) (Table 2).

Tab. 2 Comparison of p-selectin expression in untreated and treated patients

\begin{tabular}{|l|c|c|c|c|}
\hline & $\begin{array}{c}\text { Untreated } \\
\text { patients }\end{array}$ & $\begin{array}{c}\text { Treated } \\
\text { patients }\end{array}$ & $\begin{array}{c}\text { Fisher exact test } \\
\text { (p-value) }\end{array}$ & OR + 95 \% CI \\
\hline $\begin{array}{l}\text { Patients with increased } \\
\text { p-selectin expression (n) }\end{array}$ & $10 / 12$ & $26 / 43$ & 0.1830 & $\begin{array}{c}3.2692 \\
(0.6363-16.7970)\end{array}$ \\
\hline
\end{tabular}


We found that in the group of patients with lacunar stroke an increased p-selectin expression was present in 18 of 28 patients (64\%). Just a few more patients with atherothrombotic stroke had increased expression of p-selectin 19 of 27 patients (70 \%). The difference between these two groups was not statisticaly significant (p-value $=0.7753$; OR $+95 \%$ CI $=0.7579(0.2445-2.3494))($ Table 3$)$.

Tab. 3 Comparison of p-selectin expression in lacunar and atherothrombotic stroke

\begin{tabular}{|l|c|c|c|c|}
\hline & $\begin{array}{c}\text { Lacunar } \\
\text { stroke }\end{array}$ & $\begin{array}{c}\text { Atherothrombotic } \\
\text { stroke }\end{array}$ & $\begin{array}{c}\text { Fisher exact test } \\
\text { (p-value) }\end{array}$ & OR + 95 \% CI \\
\hline $\begin{array}{l}\text { Patients with increased } \\
\text { p-selectin expression (n) }\end{array}$ & $18 / 28$ & $19 / 27$ & 0.7753 & $\begin{array}{c}0.7579 \\
(0.2445-2.3494)\end{array}$ \\
\hline
\end{tabular}

\section{DISCUSSION}

The increased risk of stroke may be related to the prothrombotic or hypercoagulable state with abnormalities of hemostasis including platelet activation. The participation of activated platelets in the development of focal ischemia was already suspected in the 1960s (9).

Our study showed excessed platelet p-selectin activation during the acute phase of ischemic stroke compared to healthy volunteers and is consistent with up-to date studies which also report increased platelet p-selectin activation in patients with acute brain ischemia $(3,5,6,7,8,9)$. Marguardt et al. showed that CD62 expression declines during the first weeks after stroke, whereas CD63 expression remains increased for at least 3 months after stroke. Therefore, CD63 could be an interesting parameter for future prospective studies that evaluate the role of platelet activation markers as potential predictors of first or recurrent ischemic events (6). Obversely, Yamazaki et al. showed increased p-selectin expression 1 month after stroke, however, a serial follow-up was not performed in their study (17). Tsai et al. found that ischemic stroke patients had significantly increased circulating CD62P, CD63, and CD40L in the acute stage compared with the control group (9).

We did not find a significant influence of antiplatelet medication on baseline p-selectin expression. The number of patients with increased p-selectin expression tended to be higher in the group of untreated patients than in the group of treated patients. These findings showed that platelet inhibitors were not associated with lower platelet p-selectin expression. They are consistent with previous results - aspirin does not modify the excretion of granules $(7,20,21)$. Yamazaki et al. compared p-selectin expression among patients treated with different antiplatelet regimens. In atherothrombotic stroke the patients treated with ticlopidine had significantly lower percentage of p-selectin expression than untreated patients $(\mathrm{p}<0.01)$. The patients treated with aspirin did not show any significant difference in p-selectin expression compared with the untreated patients. They also found that in lacunar stroke the percentages of $\mathrm{p}$-selectin expression were not significantly different among the different antiplatelet regimens. The percentage of p-selectin expression tended to be lower in patients treated with combination of aspirin and ticlopidine than in those treated with aspirin or without antiplatelet agents (17).

However, we did not assess stimulated p-selectin expression. According to several authors the measurement of platelet function assessed using p-selectin expression provides information on the effect of antiplatelet therapy $(22,23)$ and in a comparison with light transmission aggregometry, Multiplate, VerifyNow, and VASP, the p-selectin based aspirin and clopidogrel tests were at least as effective in determining the inhibitory effects of antiplatelet therapies including combined aspirin and clopidogrel, and combined aspirin and prasugrel (24). The effect of aspirin and clopidogrel on platelet expression of p-selectin was assessed 
in the large trial - Triple Antiplatelets for Reducing Dependency after Ischaemic Stroke (TARDIS) trial. TARDIS compared the effect of intensive (combined aspirin, clopidogrel, and dipyridamole) versus guideline (combined aspirin and dipyridamole, or clopidogrel alone) in patients with acute stroke or transient ischemic attack. Among patients with acute noncardioembolic ischemic stroke or TIA a regimen of intensive antiplatelet therapy for 30 days did not reduce stroke recurrence or its severity when compared with guideline antiplatelet therapy with either clopidogrel alone or combined aspirin and dipyridamole. The more aggressive strategy resulted in significantly higher and more severe bleeding rates. $(25,26)$. Bath et al. performed a substudy of TARDIS; the aim of this substudy was to assess the effect of antiplatelet agents taken at baseline on platelet function reactivity and activation. They found that aspirin and clopidogrel suppress stimulated platelet p-selectin, although one-quarter of patients on clopidogrel have high on-treatment platelet reactivity. $(11,27)$. Heptinstall et al. also demonstrated a good inhibition of AA-induced platelet reactivity in patients with acute stroke taking aspirin, but variable ADP-induced platelet reactivity in the same patients taking clopidogrel (28). According to study of Thomas et al. p-selectin test results correlated with light transmission aggregometry. Using the aspirin p-selectin test only two patients demonstrated high on-treatment platelet reactivity. This study suggests that a P2Y(12) p-selectin test is capable of detecting high on-treatment platelet reactivity, which is associated with subsequent cardiovascular events (29).

Regarding subtype of the stroke the expression of p-selectin in patients with lacunar and atherothrombotic stroke was not significantly different. These results did not support the observation of the Yamazakies et al. study. They found that the percentage of platelets expressing p-selectin was significantly higher in the patients with cardioembolic stroke and atherothrombotic stroke compared to those with lacunar stroke ( $<$ 0.05) (17). However, we excluded patients with cardioembolic stroke because of different etiology of stroke. On the other hand, our findings are consistent with the study of Mohamed et al. They found that CD62 expression was not influenced by the stroke pathophysiology (7).

Flow cytometry is an advantageous technique for the analysis of platelet receptors (30). Measurement of secretory activity through the appearance of the activation marker p-selectin on platelets now provides an alternative approach, which enables remote testing (31). Flow cytometry offers several advantages over other clinical tests of platelet function including relative simplicity, minimal manipulation of samples, preventing artifactual in vitro platelet activation, analysis in the physiological milieu of whole blood, high sensitivity for the detection of platelet subpopulations and the need for only minimal blood volumes. A theoretical disadvantage of flow cytometry relates to the possibility that activated platelets may display a decreased survival in circulation resulting in modest or nonevident platelet activation by flow cytometric analysis $(32,33)$. However, convincing experimental data support that activated platelets continue to circulate. $(34,35)$. We found good sensitivity of the assessment of CD62 as platelet activation marker by whole blood flow cytometry.

\section{CONCLUSIONS}

We demonstrated significantly higher platelet activation in patients with non-cardioembolic stroke compared to control subjects in their natural environment where the whole blood is analyzed immediately after collection without adding physiological agonists. The expression of CD62P on the platelet surface reflects cell degranulation and ,therefore, the measurement of its level is a useful tool to monitor the activation status of platelets in vivo. Antiplatelet medication - ASA and CLP supressed baseline p-selectin expression only minimally. However, we did not assess p-selectin ex vivo - stimulated expression. We did not find relationship between CD62 expression and type of stroke. 


\section{REFERENCES}

1. Smith N, Pathansali R, Bath P. Platelets and stroke. Vascular Medicine 1999; 4: 165-172.

2. Toghi H, Suzuki H, Tamura K, Kimura B. Platelet volume, aggregation, and adenosine triphosphate release in cerebral thrombosis. Stroke 1991; 22: 17-22.

3. Bath PM, Blann A, Smith N, Butterworth RJ. Von Willebrand factor, P-selectin and fibrinogen levels in patients with acute ischaemic and haemorrhagic stroke, and their relationship wit $\mathrm{h}$ stroke sub-type and functional outcome. Platelets 1998; 9, 155-159.

4. Mohr JP, Sacco RL. Classification of ischemic strokes. Stroke: pathophysiology, diagnosis, and management 1992; vol. 2. 2nd ed. $271-83$.

5. Grau A, Ruf A, Vogt A, Lichy C, Buggle F, et al. Increased fraction of circulating activated platelets in acute and previous cerebrovascular ischemia. Thromb Haemost 1998; 80:298-301.

6. Marquardt L, Ruf A, Mansmann U, Winter R, Schuler M, Buggle F, Mayer H, Grau AJ. Course of platelet activation markers after ischemic stroke. Stroke 2002;33:2570-2574.

7. Mohamed Y, El-Senousy, Ehab A. El-Seidy, Mohamed K. Khalil, Hazem A. Fayed, et al. Platelet Activation Markers in Ischemic Cerebral Stroke. Egyptian Journal of Neurology, Psychiatry and Neurosurgery 2012; 49(2):109-115.

8. Zeller JA, Tschoepe D, Kessler C. Circulating platelets show increased activation in patients with acute cerebral ischemia. Thromb Haemost 1999; 81: 373-377.

9. Tsai NW, Chang WN, Shaw CF, Jan CR, Chang HW, Huang CR, Chen SD, Chuang YC, Lee LH, Lu CH. Serial change in platelet activation markers with aspirin and clopidogrel after acute ischemic stroke. Clin Neuropharmacol. 2010 Jan-Feb;33(1):40-5.

10 Kappelmayer J, Nagy B, Miszti-Blasius K, Hevessy Zs, Setiadi H. Review: The emerging value of P-selectin as a disease marker. Clin Chem Lab Med 2004;42(5):475-486.

11. Bath PM, May J, Flaherty K, Woodhouse LJ. Remote Assessment of Platelet Function in Patients with Acute Stroke or Transient Ischaemic Attack. Stroke Research and Treatment 2017; Volume 2017, 13 pages.

12. Harrison P, Cramer EM. Platelet $\alpha$-granules. Blood Rev 1993; 7: 2-62.

13. Lee YJ, Jy W, Horstmann LL, Janania J, Reyes Y, et al. Elevated platelet microparticles in transient ischemic attacks, lacunar infarcts and multiple infarct dementias.Thromb Res 1993;72: 295-304.

14. Michelson AD. Flow cytometry: a clinical test of platelet function. Blood 1996;87:4925 - 36 .

15. Minamino T, Kitakaze M, Sanada S, Asanuma H, Kurotobi T, et al. Increased expression of P-selectin on platelets is a risk factor for silent cerebral infarction in patients with atrial fibrillation: role of nitric oxide. Circulation 1998;98:1721-27.

16. Shattil SJ, Cunningham M, Hoxie JA. Detection of activated platelets in whole blood using activation-dependent monoclonal antibodies and flow cytometry. Blood 1987;70:307 -

17. Yamazaki M, Uchiyama S, Iwata M. Measurement of platelet fibrinogen binding and p-selectin expression by flow cytometry in patients with cerebral infarction. Thromb Res 2001; 104: 197-205.

18. Nagy B, Debreceni IB, Kappelmayer J. Flow Cytometric Investigation of Classical and Alternative Platelet Activation Markers. The Journal of the International Federation of Clinical Chemistry and Laboratory Medicine 2013 Jan; 23(4): 124-134.

19. Bath PM, May J, Heptinstall S.Clinical utility of remote platelet function measurement using P-selectin: assessment of aspirin, clopidogrel, and prasugrel and bleeding disorders. Platelets. 2018 Apr 18:1-6.

20. Chronos NAF, Wilson DJ, Janes SL, Hutton RA, Büller NP, Goodall AH. Aspirin does not affect the flow cytometric detection of fibrinogen binding to, or release of -granules or lysosomes from, human platelets. Clin Sci 1994; 87: 575-580.

21. Gralnick HR, Vail M, Williams S, McKeown L, Shafer B, Hansmann K. Platelet alpha granule release is not inhibited by aspirin and is mediated by myosin light chain kinase and protein C kinase. Blood 1990; 75: 128-38. 
22. Dovlatova N, Heptinstall S, May J, Bath P. Remote platelet function testing using platelet p-selectin as a marker of platelet activation.Findings in patients with stroke or tia treated with clopidogrel. Res Pract Thromb Haemostasis.2017;1:S1.

23. Fox SC, May JA, Johnson A, et al. Effects on platelet function of an EP3 receptor antagonist used alone and in combination with a $\mathrm{P}_{12} \mathrm{Y}_{12}$ antagonist both in-vitro and ex-vivo in human volunteers. Platelets. 2013;24(5):392-400.

24. Fox SC, May JA, Dovlatova N, Glenn JR, Johnson A, White AE,Radhakrishnan A, Heptinstall S. How does measurement of platelet p-selectin compare with other methods of measuring platelet function as a means of determining the effectiveness of antiplatelet therapy? Platelets. 2018;1-6

25. Bath PM, Woodhouse LJ, Appleton JP, Beridze M, Christensen H,Dineen RA, Duley L, England TJ, Flaherty K, Havard D, et al. Intensive versus guideline antiplatelet therapy in patients with acute cerebral ischaemia (tardis): a randomised, open-label, phase iii,superiority trial. Lancet. 2018;391:850859.

26. TARDIS Trial Investigators, Krishnan K, Beridze M, Christensen H,Dineen R, Duley L, Heptinstall S, James M, Markus H, Pocock S, et al. Safety and efficacy of intensive vs. Guideline antiplatelet therapyin high-risk patients with recent ischemic stroke or transient ischemic attack: rationale and design of the triple antiplatelets for reducing dependency after ischaemic stroke (tardis) trial(isrctn47823388). Int J Stroke. 2015;10:1159-1165.

27. Heptinstall S, Dovlatova N, May J, Robson K, Bath P. Remote platelet function testing using measurements of p-selectin in patients with acute stroke or tia already receiving treatment with antiplatelet agents. J Thromb Haemostasis. 2015;13:695.

28. Heptinstall S. Remote platelet function testing using platelet-bound p-selectin as a marker of platelet activation. Res Pract Thromb Haemost.2017;1:S1

29. Thomas MR, Wijeyeratne YD, May JA, Johnson A, Heptinstall S, Fox SC. A platelet P-selectin test predicts adverse cardiovascular events in patients with acute coronary syndromes treated with aspirin and clopidogrel. Platelets 2014;25:612-618.

30. Johnston GI, Pickett EB, McEver RP, George JN. Heterogeneity of platelet secretion in response to thrombin demonstrated by flow cytometry. Blood 1987;69:1401-3.

31. Algahtani M, Heptinstall S. Novel strategies for assessing platelet reactivity. Future Cardiol. 2017 Jan;13(1):33-47.

32. Rinder HM, Murphy M, Mitchell JG, Stocks J, Ault KA, Hillman RS. Progressive platelet activation with storage:evidence for shortened survival of activated platelets after transfusion. Transfusion 1991;31:409-14.

33. Sirolli V, Ballone E, Garofalo D, Merciaro G, Settefrati N, Di Mascio R, et al. Platelet activation markers in patients with nephrotic syndrome. A comparative study of different platelet function tests. Nephron 2002;91:424-30.

34. Berger G, Hartwell DW, Wagner DD. P-selectin and platelet clearance. Blood 1998;92:4446-52.

35. Michelson AD, Bernard MR, Hechtman HB, MacGragor H, Connolly RJ, Loscalzol J, et al. In vivo tracking of platelets: circulating degranulated platelets rapidly lose surface P-selectin but continue to circulate and function.Proc Natl Acad Sci USA 1996;93:11 877-82.

Received: May, 2, 2018

Accepted: June, 7, 2018 